ANNALES

POLONICI MATHEMATICI

$82.3(2003)$

\title{
A class of functions containing polyharmonic functions in $\mathbb{R}^{n}$
}

by V. Anandam and M. Damlakhi (Riyadh)

Abstract. Some properties of the functions of the form $v(x)=\sum_{i=0}^{m}|x|^{i} h_{i}(x)$ in $\mathbb{R}^{n}$, $n \geq 2$, where each $h_{i}$ is a harmonic function defined outside a compact set, are obtained using the harmonic measures.

1. Introduction. Let $\Omega$ be a clamped plate with an external load density $f(x), x \in \Omega$. If $\partial \Omega$ is regular for the Dirichlet problem, the solution $u(x)$ corresponding to the conditions of elasticity given by $\Delta^{2} u(x)=f(x)$ on $\Omega, u=0=\partial u / \partial n$ on $\partial \Omega$, has a representation (Nicolesco [16, p. 40]) $u(x)=\int_{\Omega} G_{2}(x, y) f(y) d y$. However, the calculation of $G_{2}(x, y)$ corresponding to a given $\Omega$ is not simple.

This note, among other results, shows that in the particular case of $\Omega$ being in the form of a star domain with centre 0 and the equation $\Delta^{2} u=f$ being reduced to the condition $\Delta u(x)=G(x)+|x|^{-1} H(x)$ where $G$ and $H$ are harmonic functions on $\Omega$, continuous on $\bar{\Omega}$, we can express $u$ as $u(x)=|x|^{2} h_{2}(x)+|x| h_{1}(x)+h_{0}(x)$ where $h_{i}(x)$ are harmonic functions on $\Omega$. This suggests the study of the properties of functions of the form $u(x)=\sum_{i=0}^{m}|x|^{i} h_{i}(x)$ in a star domain $\Omega$ with centre 0 in $\mathbb{R}^{n}, n \geq 2$, which include the polyharmonic functions of finite order on $\Omega$.

In another context, when $u(x)=\sum_{i=0}^{m}|x|^{i} h_{i}(x)$ is defined on the whole of $\mathbb{R}^{n}, n \geq 2$, where each $h_{i}$ is harmonic on $\mathbb{R}^{n}$, Nakai and Tada ([15, Theorem 3]) give a necessary and sufficient condition on $u$ so that each $h_{i}$ is a harmonic polynomial, by using the Fourier expansion method. In this note we obtain some complementary results for such functions $u$, by using harmonic measures.

2. Functions in the class $H^{*}\left(\mathbb{R}^{n}\right)$. We begin with the following definition.

2000 Mathematics Subject Classification: Primary 31B30.

Key words and phrases: harmonic measure, polyharmonic functions, Liouville theorem. 
Definition 2.1. Let $\Omega$ be a star domain with centre 0 in $\mathbb{R}^{n}, n \geq 2$. A continuous function $u(x)$ defined on $\Omega$ is said to be in the class $H^{m}(\Omega)$ if it is of the form $u(x)=\sum_{i=0}^{m}|x|^{i} h_{i}(x)$ where $h_{i}, 0 \leq i \leq m$, are harmonic on $\Omega$. Write $H^{*}(\Omega)=\bigcup_{m \geq 0} H^{m}(\Omega)$.

We remark that if $u(x)$ is a polyharmonic function of order $m$ on $\Omega$ (that is, $u \in C^{2 m}(\Omega)$ and $\Delta^{m} u=0$ ), then the Almansi representation of $u$ (see Aronszajn et al. [8, Proposition 1.3]) is of the form $u(x)=\sum_{i=0}^{m-1}|x|^{2 i} h_{i}(x)$ where $h_{i}(x)$ are uniquely determined harmonic functions on $\Omega$. Hence $u \in$ $H^{2 m-2}(\Omega), H^{0}(\Omega)$ being the class of all harmonic functions on $\Omega$.

In this section, we obtain some Liouville-type theorems for functions in $H^{*}\left(\mathbb{R}^{n}\right)$. For a given continuous function $f$ on $\mathbb{R}^{n}$, let $D_{r} f$ stand for the Dirichlet solution in $|x|<r$ with boundary value $f(x)$ on $|x|=r$.

TheORem 2.2. Let $u \in H^{*}\left(\mathbb{R}^{n}\right)$. Let $a_{j}$ be a sequence of real numbers increasing to $\infty$. Let $\omega$ be a nonempty open set and e be a polar set in $\mathbb{R}^{n}$. If

$$
\liminf _{j \rightarrow \infty} \frac{D_{a_{j}} u(z)}{a_{j}}=0
$$

for every $z \in \omega \backslash e$, then $u$ is harmonic on $\mathbb{R}^{n}$.

Proof. Let $u(x)=\sum_{i=0}^{m}|x|^{i} h_{i}(x)$. Then, for fixed $z \in \omega \backslash e$ and large $j$, $D_{a_{j}} u(z)=\sum_{i=0}^{m} a_{j}^{i} h_{i}(z)$. Hence the assumption on $D_{a_{j}} u$ as $j \rightarrow \infty$ implies that $h_{i}(z)=0$ for $1 \leq i \leq m$. Since $z$ is arbitrary in $\omega \backslash e, h_{i}$ vanishes on $\omega \backslash e$, which implies that $h_{i} \equiv 0$ for $1 \leq i \leq m$. Consequently, $u(x)=h_{0}(x)$ is harmonic on $\mathbb{R}^{n}$.

Corollary 1 (see [15, Proposition 1]). Let $u=\sum_{i=0}^{m}|x|^{i} h_{i}(x) \in H^{*}\left(\mathbb{R}^{n}\right)$. If $u(x) \rightarrow 0$ when $|x| \rightarrow \infty$, in particular if $u \equiv 0$, then each $h_{i} \equiv 0$.

Proof. As in the proof of Theorem 2.2 we can show that $h_{i} \equiv 0$ for $1 \leq i \leq m$ and $u=h_{0}$ on $\mathbb{R}^{n}$. Since $u \rightarrow 0$ at infinity, $h_{0} \equiv 0$.

Corollary 2. Let $u \in H^{*}\left(\mathbb{R}^{n}\right)$. Let $M(r,|u|)$ denote the mean value of $|u(x)|$ on $|x|=r$. Suppose $M(r,|u|)=o(r)$ as $r \rightarrow \infty$. Then $u$ is a constant.

Proof. Write $D_{r} u(z)=\int u(x) d \varrho_{z}^{r}(x)$ where $\varrho_{z}^{r}$ is the harmonic measure on $|x|=r$. Recall

$$
d \varrho_{z}^{r}(x)=r^{n-2} \frac{r^{2}-|z|^{2}}{|x-z|^{n}} d \sigma_{r}(x)
$$

where $d \sigma_{r}(x)$ is the measure on $|x|=r$, invariant with respect to the rotations of $|x|=r$ and such that $\sigma_{r}(|x|=r)=1$. Suppose now $|z| \leq 1<r$ $=|x|$. Then

$$
\frac{r^{2}-|z|^{2}}{|x-z|^{n}} \leq \frac{r^{2}}{(r-1)^{n}}
$$


so that

$$
\left|D_{r} u(z)\right| \leq \int|u(x)| \frac{r^{n}}{(r-1)^{n}} d \sigma_{r}(x) .
$$

Consequently, for large $r,\left|D_{r} u(z)\right| \leq 2 M(r,|u|)$ so that $\lim _{r \rightarrow \infty} D_{r} u(z) / r$ $=0$ for $|z| \leq 1$. Then by the above theorem, we conclude that $u$ is harmonic on $\mathbb{R}^{n}$; this, together with the condition that $M(r,|u|)=o(r)$, implies that $u$ is a constant. (See [6, Corollary 3.3] for an indication of different proofs of this result.)

COROLlary 3. Let $u \in H^{*}\left(\mathbb{R}^{n}\right)$ be such that for a superharmonic function $s$ on $\mathbb{R}^{n},|u| \leq s$. Then $u$ is a constant.

Proof. We write $s=p+c$ where $p \geq 0$ is a potential on $\mathbb{R}^{n}(p \equiv 0$ is the only potential on $\mathbb{R}^{2}$ ) and $c \geq 0$ is a constant. Since $M(r, p) \rightarrow 0$ as $r \rightarrow \infty$, $M(r,|u|)=o(r)$. Hence by Corollary $2, u$ is harmonic on $\mathbb{R}^{n}$ and $|u| \leq p+c$, which implies that $|u| \leq c$ on $\mathbb{R}^{n}$ so that $u$ is a constant.

REMARK. The above Corollary 3 expresses the classical Liouville-Picard theorem for the class $H^{*}\left(\mathbb{R}^{n}\right)$. In fact, in its standard form this theorem states that if $u \in H^{0}\left(\mathbb{R}^{n}\right)$ and $u \geq 0$, then it is a constant; this can be generalized as follows: If $u \in H^{*}\left(\mathbb{R}^{n}\right)$ is a positive superharmonic function, then $u$ is a constant. This generalization is a consequence of the above Corollary 3. However, if we leave out the condition that $u$ is superharmonic, we have from Armitage [7] or Futamura-Kishi-Mizuta [13] or Nakai-Tada [15] the following: If $u \in H^{m}\left(\mathbb{R}^{n}\right)$ is positive, then $u$ is a polynomial of degree at most $m$. More generally, let $u(x)=\sum_{i=0}^{m}|x|^{i} h_{i}(x)$ and suppose $\liminf \operatorname{in}_{|x| \rightarrow \infty} u(x) /|x|^{s} \geq 0$ for some $s>m$. Then for each $i, h_{i}$ is a harmonic polynomial of degree less than $s-i$. For as in [6, Lemma 2.1] we can find a locally integrable function $\varphi(x)$ on $\mathbb{R}^{n}$ such that $u(x) \geq \varphi(x)$ outside a compact set $K$ and $M(r,|\varphi|)=o\left(r^{s}\right)$ as $r \rightarrow \infty$. Since $u \geq-|\varphi|$ on $\mathbb{R}^{n} \backslash K$, $M\left(r, u^{-}\right)=o\left(r^{s}\right)$ as $r \rightarrow \infty$; also $M(r, u)=O\left(r^{m}\right)$. Hence $M(r,|u|)=o\left(r^{s}\right)$. Consequently, each $h_{i}$ is a harmonic polynomial of degree less than $s-i$. In particular, if $\liminf |x| \rightarrow \infty u(x) /|x| \geq 0$ then $u$ is a constant.

3. $H^{*}$ functions on a star domain. Let $\Omega$ denote a star domain in $\mathbb{R}^{n}$, $n \geq 2$, with centre 0. Then, as in Definition 2.1, $H^{m}(\Omega)$ denotes the class of functions of the form $u(x)=\sum_{i=0}^{m}|x|^{i} h_{i}(x)$ where $h_{i}$ are harmonic on $\Omega$. We shall use the operator $\Delta^{p}$, with integer $p \geq 1$, in the sense of distributions.

LEMma 3.1. If $u \in C^{2 p}(\Omega)$, let $f(x)=|x|^{\alpha} u(x)$ where $\alpha>2 p-n$. Then $\Delta^{p}\left(|x|^{\alpha} u(x)\right)$ is locally integrable on $\Omega$.

Proof. Since $(\partial / \partial x)^{\lambda}|x|^{\alpha}$ is locally integrable on $\mathbb{R}^{n}$ when $\alpha>|\lambda|-n$, the lemma is evident. We need the following expression of $\Delta^{p} f(x)$ for later use. 
For $|x|=r>0$, let $g=\Delta^{p} f$ in the classical sense.

Since

$$
\Delta f=\alpha(\alpha+n-2) r^{\alpha-2} u+2 \alpha r^{\alpha-2} \sum x_{i} \frac{\partial u}{\partial x_{i}}+r^{\alpha} \Delta u,
$$

we have $\Delta f=r^{\alpha-2} u_{1}$ where $u_{1} \in C^{2 p-2}$ (note that $u_{1}$ is harmonic if $u$ is). Proceeding thus, we find $\Delta^{j} f=r^{\alpha-2 j} u_{j}$ for $1 \leq j \leq p$, where $u_{j} \in C^{2 p-2 j}$. Let $g(x)=|x|^{\alpha-2 p} u_{p}(x)$ for $|x|>0$ and $g(0)=\limsup _{x \rightarrow 0} g(x)$. Then $g(x)$ is u.s.c. on $\Omega$ and since $\alpha-2 p+n>0, g(x)$ is locally integrable on $\Omega$ and $\Delta^{p} f=g$ on $\Omega$ in the sense of distributions.

REMARK. Let $h$ be harmonic on $\Omega$. Then from the above proof (replacing $u$ by $h)$ we see that if $\alpha>2 p-n$, then $\Delta^{p}\left(|x|^{\alpha} h(x)\right)=|x|^{\alpha-2 p} H(x)$ where $H(x)$ is harmonic on $\Omega$.

Proposition 3.2. Let $u \in H^{*}(\Omega)$ and $2 p \leq n$. Then $\Delta^{p} u$ is locally integrable on $\Omega$.

Proof. Let $u=\sum_{i=0}^{m}|x|^{i} h_{i}(x)$. Then for any $i \geq 1, i>2 p-n$ so that $\Delta^{p}\left(|x|^{i} h_{i}(x)\right)$ is locally integrable on $\Omega$; for $i=0, \Delta\left(h_{0}\right)=0$. Hence $\Delta^{p} u$ is locally integrable on $\Omega$.

Consequence. The above proposition, in particular, states that if $u \in$ $H^{*}\left(\mathbb{R}^{n}\right), n \geq 2$, then $\Delta u$ is locally integrable on $\mathbb{R}^{n}$. This leads to an integral representation of $u$ in $\mathbb{R}^{n}$. For that, recall that given any positive Radon measure $\mu$ on an open set $\omega$ in $\mathbb{R}^{n}, n \geq 2$, Brelot [12] shows that a subharmonic function $s$ can be constructed on $\omega$ with associated measure $\mu$ in the local Riesz representation.

Now, for $u \in H^{*}\left(\mathbb{R}^{n}\right), n \geq 2$, since $\Delta u$ is locally integrable, $d \lambda(x)=$ $\Delta u d x$ can be treated as defining the difference of two positive Radon measures on $\mathbb{R}^{n}$. Hence $u$ is the difference of two subharmonic functions on $\mathbb{R}^{n}$. Then we can define the order of $u$ and the order of $\lambda$ as in Arsove [9] (see also [4]). If the order of $\lambda$ is finite, a correspondingly modified form of the logarithmic kernel (if $n=2$ ) or the Newtonian kernel (if $n \geq 3$ ) can be used to represent $u$ as an integral up to an additive harmonic function which is a harmonic polynomial if the order of $u$ is finite (see Arsove [9], and [4, Theorems 11 and 12]; see also Mizuta [14]).

Lemma 3.3. Let $H$ be a harmonic function on $\Omega$. If $n+\alpha-2>0$ and if $\alpha+2 i \neq 0$ for $i, 0 \leq i \leq p-1$, then there exists a harmonic function $h$ on $\Omega$ such that $\Delta^{p}\left(|x|^{\alpha+2 p-2} h(x)\right)=|x|^{\alpha-2} H(x)$.

Proof. We prove the lemma for the case $p=1$ by adapting the method given in Aronszajn et al. [8, p. 5]. The general case follows by induction. Suppose a harmonic function $h$ exists on $\Omega$ such that $\Delta\left(|x|^{\alpha} h(x)\right)=|x|^{\alpha-2} H(x)$. 
Then, treating $h$ as a function of $r$, we should have

$$
\Delta\left(r^{\alpha} h\right)=\alpha(n+\alpha-2) r^{\alpha-2} h+2 \alpha r^{\alpha-1} \frac{\partial h}{\partial r} .
$$

Then

$$
\alpha(n+\alpha-2) h+2 \alpha r \frac{\partial h}{\partial r}=H \quad \text { on } \Omega .
$$

This can be written as

$$
\frac{d}{d r}\left[r^{(n+\alpha-2) / 2} h\right]=\frac{H}{2 \alpha} r^{(n+\alpha-4) / 2}
$$

outside 0 . Since at the origin, $r^{(n+\alpha-2) / 2} h=0$, we should have

$$
r^{(n+\alpha-2) / 2} h(r, w)=\int_{0}^{r} \frac{1}{2 \alpha} \varrho^{(n+\alpha-4) / 2} H(\varrho, w) d \varrho,
$$

where $x=(r, w)$ is represented by the spherical polar coordinates. Set $\varrho=t r$. Then

$$
h(x)=\frac{1}{2 \alpha} \int_{0}^{1} t^{(n+\alpha-4) / 2} H(t x) d t
$$

here $\Delta h=0$ since $H$ is harmonic. Consequently, given the harmonic function $H$ on $\Omega$, if we define $h(x)$ by the formula above, then $h(x)$ is harmonic on $\Omega$, satisfying the condition $\Delta\left(|x|^{\alpha} h(x)\right)=|x|^{\alpha-2} H(x)$.

THEOREM 3.4. A continuous function $u$ on $\Omega$ is in $H^{m}(\Omega)$ if and only if for any integer $p, 2 \leq 2 p \leq n$, there exists a function $v \in H^{m-1}(\Omega)$ such that $\Delta^{p} u(x)=|x|^{1-2 p} v(x)$ in the sense of distributions.

Proof. (1) Let $u=\sum_{i=0}^{m}|x|^{i} h_{i} \in H^{m}(\Omega)$. We shall now use the Remark following Lemma 3.1 to calculate $\Delta^{p} u(x)$.

If $p=1$, then $\Delta u=\sum_{i=0}^{m-2}|x|^{i} H_{i}+|x|^{-1} v_{1}$ where $H_{i}(0 \leq i \leq m-2)$ and $v_{1}$ are harmonic functions on $\Omega$. Hence $\Delta u=|x|^{-1}\left[\sum_{i=0}^{m-2}|x|^{i+1} H_{i}+v_{1}\right]=$ $|x|^{-1} s_{1}(x)$ where $s_{1} \in H^{m-1}(\Omega)$.

If $p=2$, then $n \geq 4$ and in this case $\Delta\left(|x|^{-1} v_{1}\right)=|x|^{-3} v_{2}$ where $v_{2}$ is harmonic on $\Omega$. This leads to the equation $\Delta^{2} u=\Delta(\Delta u)=\sum_{i=0}^{m-4}|x|^{i} H_{i}^{\prime}+$ $|x|^{-1} v+|x|^{-3} v_{2}$ on $\Omega$, where $H_{i}^{\prime}(0 \leq i \leq m-4), v$ and $v_{2}$ are harmonic on $\Omega$. This simplifies to the form $\Delta^{2} u=|x|^{-3} s_{2}(x)$ where $s_{2} \in H^{m-1}(\Omega)$.

This process by induction leads to the result that if $2 \leq 2 p \leq n$, then $\Delta^{p} u=|x|^{-(2 p-1)} s_{p}(x)$ where $s_{p} \in H^{m-1}(\Omega)$.

(2) Conversely, suppose that $u$ is a continuous function on $\Omega$ such that for any integer $p, 2 \leq 2 p \leq n$, we have $\Delta^{p} u=|x|^{-(2 p-1)} v$ where $v \in H^{m-1}(\Omega)$. Then, in particular for $p=1$, by Lemma 3.3 we have

$$
\Delta u=|x|^{-1} v=|x|^{-1} \sum_{i=0}^{m-1}|x|^{i} H_{i}=\sum_{i=0}^{m-1}|x|^{i-1} H_{i}=\sum \Delta\left(|x|^{i+1} h_{i}\right) .
$$


Hence $u=\left(\sum_{i=0}^{m-1}|x|^{i+1} h_{i}\right)+($ a harmonic function on $\Omega)$; in other words, $u \in H^{m}(\Omega)$.

Corollary 1. Let $u=\sum_{i=0}^{m}|x|^{i} h_{i}(x) \in H^{m}(\Omega)$. Suppose $u$ is harmonic on a neighbourhood of a point in $\Omega$. Then $h_{i} \equiv 0$ for $1 \leq i \leq m$. In particular, if $u \equiv 0$ on a nonempty open set in $\Omega$, then $h_{i} \equiv 0$ for all $i$, $0 \leq i \leq m$.

Proof. Let $u$ be harmonic on a nonempty open set $\omega$. Since $u \in H^{m}(\Omega)$, there exists $v_{m-1} \in H^{m-1}(\Omega)$ such that $\Delta u=|x|^{-1} v_{m-1}(x)$ on $\Omega$. This implies $v_{m-1}=0$ on $\omega$. Now again by Theorem 3.4, there exists $v_{m-2} \in$ $H^{m-2}(\Omega)$ such that $\Delta v_{m-1}=|x|^{-1} v_{m-2}(x)$, which implies that $v_{m-2}=0$ on $\omega$. Proceeding thus, we obtain $v_{i} \in H^{i}(\Omega), 0 \leq i \leq m-1$, such that $\Delta v_{i+1}=|x|^{-1} v_{i}$ on $\Omega$ and $v_{i}=0$ on $\omega$ (taking $v_{m}=u$ ).

Since $v_{0}$ is harmonic on $\Omega$ and $v_{0}=0$ on $\omega, v_{0} \equiv 0$ on $\Omega$. This implies $v_{1}$ is harmonic on $\Omega$ and since $v_{1}=0$ on $\omega$, we have $v_{1} \equiv 0$ on $\Omega$. Thus proceeding, we remark that $v_{i} \equiv 0$ on $\Omega$ for $0 \leq i \leq m-1$ so that $\Delta u=0$ on $\Omega$; that is, $u$ is harmonic on $\Omega$.

Then $\sum_{i=1}^{m}|x|^{i} h_{i}(x)=u(x)-h_{0}(x)$ is harmonic on $\Omega$. Choose $a$ such that $\{x:|x|<a\} \subset \Omega$. Fix $z \in \Omega$ so that $|z|<r<a$. Let $\varrho_{z}^{r}$ be the harmonic measure on $|x|=r$. Then

$$
\sum_{i=1}^{m} \int|x|^{i} h_{i}(x) d \varrho_{z}^{r}(x)=\int\left(u-h_{0}\right) d \varrho_{z}^{r},
$$

which implies that

$$
\sum_{i=1}^{m} r^{i} h_{i}(z)=u(z)-h_{0}(z) .
$$

Since $r$ is arbitrary in the interval $(|z|, a)$, we have $h_{i}(z)=0$ for $1 \leq i \leq m$ and $u(z)-h_{0}(z)=0$. Since $h_{i}$ and $u$ are harmonic on $\Omega$ and $z$ is arbitrary except for the condition $|z|<a$, we conclude $h_{i} \equiv 0$ on $\Omega$ for $1 \leq i \leq m$ and $u \equiv h_{0}$.

Corollary 2. Let $u \in H^{m}(\Omega)$ and $2 \leq 2 p \leq n$. Suppose $u$ is p-harmonic $\left(\Delta^{p} u=0\right)$ on a neighbourhood of a point in $\Omega$. Then $u$ is p-harmonic on $\Omega$.

Proof. Suppose $\Delta^{p} u=0$ on a nonempty open set $\omega$. By Theorem 3.4, there exists a function $v \in H^{m-1}(\Omega)$ such that $\Delta^{p} u=|x|^{1-2 p} v$ on $\Omega$. Hence $v=0$ on $\omega$ and consequently, by Corollary $1, v \equiv 0$ on $\Omega$, which means that $u$ is $p$-harmonic on $\Omega$.

REMARK. We thank the referee for pointing out that in the proofs of the above two corollaries, one can use the real analyticity of $u$ outside the origin, without having recourse to Theorem 3.4 . 
Corollary 3. Let $u \in H^{m}(S)$, where $S=\{x:|x|<1\}$ in $\mathbb{R}^{n}$, be such that $\liminf _{r \rightarrow 1} M(r,|u|)=0$. Then $u(x)=(1-|x|) v(x)$ where $v \in H^{m-1}(S)$.

Proof. Let $u(x)=\sum_{i=0}^{m}|x|^{i} h_{i}(x) \in H^{m}(S)$. Notice that by the above Corollary 1, there exist uniquely determined harmonic functions $u_{i}$ on $S$ such that

$$
u(x)=\sum_{i=0}^{m}(1-|x|)^{i} u_{i}(x) \quad \text { on } S .
$$

In particular,

$$
h_{0}(x)=\sum_{i=0}^{m} u_{i}(x) .
$$

Fix $z$ with $|z| \leq 1 / 4$. For $|x|=r, 1 / 2 \leq r<1$, let $d \varrho_{z}^{r}(x)$ denote the harmonic measure on $|x|=r$. Integrate $u(x)$ with respect to $d \varrho_{z}^{r}(x)$ to get

$$
\left|(1-r)^{m} u_{m}(z)+\ldots+(1-r) u_{1}(z)+u_{0}(z)\right|=\left|\int u(z) d \varrho_{z}^{r}(x)\right| \leq C M(r,|u|)
$$

for some constant $C$ since $|z| \leq 1 / 4$ and $1 / 2 \leq r<1$ (see the proof of Corollary 2 to Theorem 2.2).

Let $r \rightarrow 1$. Since $\liminf _{r \rightarrow 1} M(r,|u|)=0$ by hypothesis, we obtain $u_{0}(z)=0$ for $|z| \leq 1 / 4$. Hence $u_{0} \equiv 0$. Consequently, $u(x)=(1-|x|) v(x)$ where

$$
v(x)=\sum_{i=0}^{m-1}(1-|x|)^{i} u_{i+1}(x) \in H^{m-1}(S) .
$$

Note. The above corollary easily includes the result: If $u$ is $p$-harmonic on $S$ such that $\liminf _{r \rightarrow 1} M(r,|u|)=0$, then $u(x)=\left(1-|x|^{2}\right) v(x)$ where $v$ is polyharmonic on $S$ of order $\leq m-1$. This in itself is a generalization of a result of Abkar and Hedenmalm [1, pp. 321-322] proved in the complex plane using the Fourier series: If $u(z)$ is biharmonic on the unit disc in the complex plane and if $M(r,|u|)=O(1-r)$ as $r \rightarrow 1$, then $u(z)=\left(1-|z|^{2}\right) h(z)$ for a harmonic function on the unit disc.

4. $H^{*}$ functions defined near infinity. It is not surprising that in many respects, the functions in $H^{2 m-2}\left(\mathbb{R}^{n}\right)$ behave near infinity like the $m$-harmonic functions (that is, the solutions of $\Delta^{m} u=0$ ) on $\mathbb{R}^{n}$. In this section, we study a class of continuous functions on $\mathbb{R}^{n}$ which are associated near infinity with the functions in $H^{2 m-2}\left(\mathbb{R}^{n}\right)$ and the fundamental solution of $\Delta^{m}$ on $\mathbb{R}^{n}$. This class contains a significant collection of functions having some nice regularity properties at infinity.

For $m \geq 1, n \geq 2$, let $e_{m}^{n}$ denote the fundamental solution of $\Delta^{m}$ on $\mathbb{R}^{n}$. We recall that 


$$
e_{m}^{n}= \begin{cases}|x|^{2 m-n} & \text { if } 2 m<n \text { or } 2 m-n \text { is odd }>0 \\ |x|^{2 m-n} \log |x| & \text { if } 2 m-n \text { is even } \geq 0\end{cases}
$$

Definition 4.1. A continuous function $v$ defined outside a compact set in $\mathbb{R}^{n}$ is said to be in the class $H_{\infty}^{2 m-2}\left(\mathbb{R}^{n}\right)$ if there exists $u \in H^{2 m-2}\left(\mathbb{R}^{n}\right)$ such that $u-v=O\left(e_{m}^{n}\right)$ near infinity.

Proposition 4.2. Let $h_{i}(0 \leq i \leq m-2)$ be arbitrary harmonic functions defined outside a compact set in $\mathbb{R}^{n}$. Then

$$
v=\sum_{i=0}^{2 m-2}|x|^{i} h_{i}(x) \in H_{\infty}^{2 m-2}\left(\mathbb{R}^{n}\right) .
$$

Proof. Recall that (see [2] or Axler et al. [10]) given a harmonic function $h$ outside a compact set in $\mathbb{R}^{n}$, there exists a harmonic function $H$ on $\mathbb{R}^{n}$ and a constant $\alpha$ such that outside a compact set,

$$
h(x)= \begin{cases}H(x)+\alpha \log |x|+g(x) & \text { if } n=2 \\ H(x)+g(x) & \text { if } n \geq 3\end{cases}
$$

where $g(x)$ is a harmonic function satisfying $g(x)=O\left(|x|^{2-n}\right)$ near infinity. Hence for each $i, 0 \leq i \leq 2 m-2$, there exists a harmonic function $H_{i}$ on $\mathbb{R}^{n}$ such that $\left|h_{i}-H_{i}\right| \leq A|x|^{2-n}$ if $n \geq 3$ and $\left|h_{i}-H_{i}-\alpha_{i} \log \right| x|| \leq A$ if $n=2$, outside a compact set. Let $u(x)=\sum_{i=0}^{2 m-2}|x|^{i} H_{i}(x)$. Then $u \in H^{2 m-2}\left(\mathbb{R}^{n}\right)$ and near infinity $u-v=O\left(e_{m}^{n}\right)$. Hence $v \in H_{\infty}^{2 m-2}\left(\mathbb{R}^{n}\right)$.

COROLlary. Let $k$ be a compact set in a star domain $\Omega$ with centre 0 . Suppose $u=\sum_{i=0}^{2 m-2}|x|^{i} h_{i}(x)$ where $h_{i}$ are harmonic on $\Omega \backslash k$. Then there exist $t \in H^{2 m-2}(\Omega)$ and $s \in H_{\infty}^{m-2}\left(\mathbb{R}^{n}\right) \cap C\left(\mathbb{R}^{n} \backslash k\right), s=O\left(e_{m}^{n}\right)$ near infinity, such that $u=s-t$ on $\Omega \backslash k$.

Proof. We know that for each $i$, there exist $s_{i} \in H^{0}\left(\mathbb{R}^{n} \backslash k\right)$ and $t_{i} \in$ $H^{0}(\Omega)$ such that $h_{i}=s_{i}-t_{i}$ on $\Omega \backslash k$ (Laurent decomposition for harmonic functions, see [2] or Axler et al. [10, pp. 171-175]).

Let now

$$
s^{1}(x)=\sum_{i=0}^{2 m-2}|x|^{i} s_{i}(x) \text { and } t^{1}(x)=\sum_{i=0}^{2 m-2}|x|^{i} t_{i}(x) .
$$

Then $u=s^{1}-t^{1}$ on $\Omega \backslash k$ where by the above proposition $s^{1}(x)$ is in the class $H_{\infty}^{2 m-2}\left(\mathbb{R}^{n}\right)$, and $t^{1}(x) \in H^{2 m-2}(\Omega)$. Since $s^{1} \in H_{\infty}^{2 m-2}\left(\mathbb{R}^{n}\right)$, there exists $v \in H^{2 m-2}\left(\mathbb{R}^{n}\right)$ such that $s^{1}-v=O\left(e_{m}^{n}\right)$ near infinity. Write now $s=s^{1}-v$ and $t=t^{1}-v$ to obtain the decomposition $u=s-t$ on $\Omega \backslash k$ as stated in the Corollary.

Proposition 4.3. If $v$ is an m-harmonic function defined outside a compact set in $\mathbb{R}^{n}$, then $v \in H_{\infty}^{2 m-2}\left(\mathbb{R}^{n}\right)$. 
Proof. For $m=1$, the representation for a harmonic function $h$ outside a compact set (given in the proof of Proposition 4.2) leads to the result that $h \in H_{\infty}^{1}\left(\mathbb{R}^{n}\right)$.

Let us take the case $m=2$. In this case, we start with the representation for a biharmonic function $b$ defined near infinity in the following form (see [11, p. 19]):

$$
b(x)= \begin{cases}\left(\alpha+\alpha_{1} x_{1}+\alpha_{2} x_{2}\right) \log |x|+\beta|x|^{2} \log |x|+B(x)+u(x) & \text { if } n=2, \\ \beta|x|+B(x)+u(x) & \text { if } n=3, \\ \beta \log |x|+B(x)+u(x) & \text { if } n=4, \\ B(x)+u(x) & \text { if } n \geq 5,\end{cases}
$$

where $B(x)$ is biharmonic on $\mathbb{R}^{n}$ and $u(x)$ is biharmonic bounded near infinity. In the case of $n \geq 5$, we can show that $|u(x)| \leq A|x|^{4-n}$ by specializing the proof $(1) \Rightarrow(2)$ of $\left[11\right.$, Theorem 10]. Consequently, since $B \in H^{2}\left(\mathbb{R}^{n}\right)$ and since $b-B=O\left(e_{2}^{n}\right)$ near infinity, we have $b \in H_{\infty}^{2}\left(\mathbb{R}^{n}\right)$.

Finally, for $m>2$, we have a similar representation for an $m$-harmonic function defined outside a compact set (details given in a forthcoming paper [5]) which can be used to prove the proposition. The result referred to here is as follows: Let $u$ be $m$-harmonic outside a compact set in $\mathbb{R}^{n}$. Then there exists an $m$-harmonic function $v$ on $\mathbb{R}^{n}$ such that $u-v=O\left(e_{m}^{n}\right)$ as $|x| \rightarrow \infty$.

TheOREM 4.4. Let $v \in H_{\infty}^{2 m-2}\left(\mathbb{R}^{n}\right), n>2 m \geq 2$. Suppose either one of the following conditions is satisfied:

(1) There exists a superharmonic function s outside a compact set such that $|v| \leq s$ near infinity.

(2) $\lim _{|x| \rightarrow \infty} v(x) /|x|=0$.

Then $\lim _{|x| \rightarrow \infty} v(x)$ exists and is finite.

Proof. (1) Suppose $|v| \leq s$ near infinity. Since $n \geq 3$, there exists a superharmonic function $S$ on $\mathbb{R}^{n}$ such that $S-s$ is bounded near infinity (see [3]). Hence we can as well assume that $s$ is a superharmonic function defined on the whole of $\mathbb{R}^{n}$ and $|v| \leq s$ near infinity.

Since $v \in H_{\infty}^{2 m-2}\left(\mathbb{R}^{n}\right)$, by definition there exists $u \in H^{2 m-2}\left(\mathbb{R}^{n}\right)$ such that $|u-v| \leq A|x|^{2 m-n}$ near infinity. Hence $|u| \leq s+A|x|^{2 m-n} \leq s+A$ near infinity. Then by Corollary 3 in Section $2, u$ is a constant $\alpha$. Consequently, $\lim _{x \rightarrow \infty} v(x)=\alpha$.

(2) Suppose now $\lim _{|x| \rightarrow \infty} v(x) /|x|=0$. Since $v \in H_{\infty}^{2 m-2}\left(\mathbb{R}^{n}\right)$, there exists $u \in H^{*}\left(\mathbb{R}^{n}\right)$ such that $|u-v| \leq A|x|^{2 m-n}$ near infinity. This implies that $\lim _{|x| \rightarrow \infty} u(x) /|x|=0$. Hence, by Corollary 2 in Section $2, u$ is a constant $\alpha$. Consequently $\lim _{|x| \rightarrow \infty} v(x)=\alpha$.

REMARK. Since every bounded continuous function $v$ is in $H_{\infty}^{2 m-2}$ if $2 \leq n \leq 2 m$, the above theorem is not valid if $n \leq 2 m$. 
We thank the College of Science Research Center, King Saud University, for the grants MATH $1419 \backslash 14$ and MATH $1420 \backslash 20$.

\section{References}

[1] A. Abkar and H. Hedenmalm, A Riesz representation formula for super-biharmonic functions, Ann. Acad. Sci. Fenn. Math. 26 (2001), 305-324.

[2] V. Anandam, Sur une propriété de la fonction harmonique, Bull. Sci. Math. 101 (1977), 255-263.

[3] -, Subharmonic functions outside a compact set in $\mathbb{R}^{n}$, Proc. Amer. Math. Soc. 84 (1982), 52-54.

[4] -, On the integral representation of biharmonic functions in $\mathbb{R}^{n}$, Ann. Acad. Sci. Fenn. Ser. A I Math. 8 (1983), 357-367.

[5] - Polysubharmonic functions near infinity, to appear.

[6] V. Anandam and M. Damlakhi, Harmonic singularity at infinity in $\mathbb{R}^{n}$, Real Anal. Exchange 23 (1997/98), 471-476.

[7] D. Armitage, A Liouville theorem for polyharmonic functions, Hiroshima Math. J. 31 (2001), 367-370.

[8] N. Aronszajn, T. M. Creese and L. J. Lipkin, Polyharmonic Functions, Clarendon Press, Oxford, 1983.

[9] M. Arsove, Functions representable as differences of subharmonic functions, Trans. Amer. Math. Soc. 75 (1953), 327-365.

[10] S. Axler, P. Bourdon and W. Ramey, Harmonic Function Theory, Springer, 1992.

[11] I. Bajunaid and V. Anandam, Biharmonic extensions in Riemannian manifolds, Hiroshima Math. J. 30 (2000), 9-20.

[12] M. Brelot, Sur l'intégration de $\Delta u(M)=\varphi(M)$, C. R. Acad. Sci. Paris 201 (1935), $1316-1318$.

[13] T. Futamura, K. Kishi and Y. Mizuta, A generalization of the Liouville theorem to polyharmonic functions, J. Math. Soc. Japan 53 (2001), 113-118.

[14] Y. Mizuta, An integral representation and fine limits at infinity for functions whose Laplacians iterated $m$ times are measures, Hiroshima Math. J. 27 (1997), 415-427.

[15] M. Nakai and T. Tada, A form of classical Liouville theorem for polyharmonic functions, ibid. 30 (2000), 205-213.

[16] M. Nicolesco, Les fonctions polyharmoniques, Hermann, Paris, 1936.

Department of Mathematics

King Saud University

P.O. Box 2455

Riyadh 11451, Saudi Arabia

E-mail: vanandam@ksu.edu.sa

damlakhi@ksu.edu.sa

Reçu par la Rédaction le 6.11.2002

Révisé le 4.6.2003 